\title{
Modelling and controller design for a UV disinfection plant
}

\author{
S. van Mourik, B.J. Geurts, H. Zwart, University of Twente, The Netherlands \\ Corresponding author: S. van Mourik \\ Department of Applied Mathematics \\ University of Twente, The Netherlands \\ Phone: +31 (53) 4893473 , Fax: +31 (53) 4893800 \\ e-mail: s.vanmourik@ewi.utwente.nl
}

\begin{abstract}
A mathematical model describing fluid flow and concentration dynamics of microorganisms inside a UV photoreactor is developed. Using physical arguments and techniques from system theory, we approximate this model by a first order linear one. For this reduced model, we design a controller. The controller is tested on the original model as well as on the reduced model by numerical simulation. This showed only very small differences in dynamics, which indicates that for the original model a classic controller with excellent properties can be designed.
\end{abstract}

\section{Introduction}

For the disinfection of fluids, UV-treatment becomes increasingly popular. This method replaces more conventional options. For example, in drinking water treatment, chlorination is still the most used disinfection method. However, since the residuals are toxic to aquatic life [20], and since some by-products of chlorination have been proven to be mutagenic, the environmentally friendly UV disinfection method has been stimulated as an alternative $[1,19]$. In $[3,4$, $17,18,21,22]$ design and modelling of a photoreactor for disinfecting drinking water is proposed. In $[12,13,15,16,23]$ experimental results are presented that link the UV dosage to the inactivation of microorganisms in wastewater treatment in agriculture and horticulture. Air disinfection by UV is investigated in [7] and [14]. In [6] the required UV dosage for apple cider pasteurization is examined, and in [5] the possibility of UV treatment of process water in the food and beverage industries is discussed.

In order to improve the operation of UV disinfection, proper dynamic control needs to be incorporated. However, surprisingly little literature is available on control design for UV disinfection. Only one approach is known to the authors. In [10] a basic model was developed, and a controller was designed. The fluid mechanics was modelled by a plug flow, and the attenuation of the microorganisms due to the UV irradiation was modelled as a first order reaction. For effective control design, it is desirable to have a low order linear model that contains the essential system dynamics. This allows for standard linear control, which is very well documented and gives generally good performance, see for example $[2,11]$. Unfortunately, models describing a UV reactor are generally not of this type. Disinfection is a complex process with often higher order reaction 
kinetics [4], and sometimes reactivation. Moreover, a detailed description of the fluid flow through the reactor is in general described by the full NavierStokes equations [8]. Altogether, a detailed reactor model leads to a nonlinear model, and hence it is not possible to design a controller in the traditional sense, employing standard techniques.

In this paper, a model-based controller is designed for a linear and low order approximation of the original model. In section 2, we describe the full transport model for the fluid and microorganism concentration, under the influence of UV irradiation. The reaction kinetics are described in a basic way, following [10]. Subsequently, we adopt the assumption of laminar flow and invoke basic symmetry properties of the solution. This yields a simplified model of which the fluid-mechanics part can be solved analytically. By specifying the model to conditions relevant to disinfection of cider [6], we obtain the reference 'nominal' model.

In section 3 the nominal model is linearized and approximated by a linear state space form by a Padé transfer function approximation. Next, the model is input/output balanced and truncated down to a first order model. In section 4 a feedback controller is designed for the resulting linear, first order model. The controller is tested by a simulation study and found to perform equally well on the reduced model as on the nominal model. This indicates that under the reduction steps, the essential dynamics of the nominal model is maintained. Hence, for the original model a classic controller with excellent properties can be designed.

\section{Basic modelling of the disinfection plant}

In this section we first sketch the general physical model. Then we proceed to analyze laminar flow in the reactor and subsequently consider the dynamics of the active microorganism concentration in case diffusive transport can be neglected. Finally, the parameter values for the nominal model are chosen, corresponding to the particular case of UV-disinfection in a cider plant.

\subsection{Physical model}

We propose a general model that describes the fluid flow and the reaction kinetics in a cylindrical reactor. This model is composed of the Navier-Stokes equations for an incompressible fluid, to describe conservation of mass and momentum, and a convection-diffusion-reaction equation which quantifies the concentration of active microorganisms in the reactor. Figure 1 shows the reactor with a UV lamp in the center along the longitudinal direction. Here, $R_{2}$ is the outer radius, $R_{1}$ the radius of the lamp, and $L^{*}$ the length of the reactor. The variables with an asterisk are scaled later on. The general equations, describing continuous fluid flow and mass transport in the reactor are (gravitational effects are neglected and the fluid is assumed to be incompressible) 


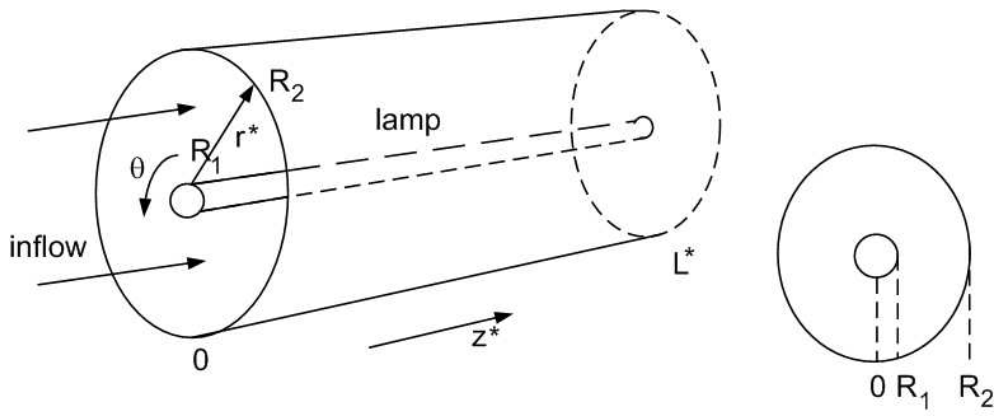

Figure 1: Schematic overview of a UV reactor.

Continuity:

$$
\nabla^{*} \cdot \mathbf{v}^{*}=0
$$

Momentum:

$$
\frac{\partial \mathbf{v}^{*}}{\partial t^{*}}=-\left(\mathbf{v}^{*} \cdot \nabla^{*}\right) \mathbf{v}^{*}-\frac{\nabla^{*} p^{*}}{\rho}+\nu \nabla^{2 *} \mathbf{v}^{*}
$$

Transport:

$$
\frac{\partial C^{*}}{\partial t^{*}}=-\left(\mathbf{v}^{*} \cdot \nabla^{*}\right) C^{*}+D \nabla^{2 *} C^{*}-K^{*} C^{*},
$$

where $\mathbf{v}^{*}$ is the velocity vector. Furthermore,

$$
D=\frac{\nu}{\operatorname{Pr}}
$$

denotes the microorganisms diffusion coefficient. $C^{*}>0$ is the concentration of microorganisms in cells per $m^{3}, \nu$ the kinematic viscosity, and $\operatorname{Pr}=\frac{\eta C_{p}}{\lambda}$ the Prandtl number, with $C_{p}$ the heat capacity, $\eta$ the viscosity, and $\lambda$ the thermal conductivity.

Finally, we consider the first order reaction mechanism as proposed in [10], using results from [3], [17], and [18]. This is described by the disinfection reaction rate

$$
K^{*}\left(r^{*}\right)=\epsilon I^{*} \frac{R_{1}}{r^{*}} \exp \left(-E^{*}\left(r^{*}-R_{1}\right)\right),
$$

which is governed by the intensity $I\left(W / m^{2}\right)$, taken as the UV intensity at the surface of the lamp at $R_{1}$, the microorganism susceptibility factor $\varepsilon\left(\mathrm{m}^{2} / J\right)$, and the monochromatic absorbance $E(1 / m)$. It is assumed that $\varepsilon$ and $E$ are constant. The most important assumptions that have been made in the derivation of (5) are

- The reaction constant only depends on $r$ and not on $z$.

- The reaction kinetics are of first order, and there is no reactivation.

- Solids are completely suspended in the medium, i.e., there are no lumps of material. 
- Reflection and refraction effects are negligible.

The boundary conditions are discussed later on.

\subsection{Scaling}

It is convenient to work with dimensionless quantities and for that purpose all variables are scaled. As reference length-scale we adopt $R_{2}$. The reference velocity is denoted by $u_{0}$ for which we select the mass-average velocity, i.e., $u_{0} A=Q$ where the reference area $A=\pi\left(R_{2}^{2}-R_{1}^{2}\right)$, and $Q$ is the volumeflux of fluid through the reactor in $m^{3} \mathrm{~s}^{-1}$. These reference scales also define a time-scale $R_{2} / u_{0}$. To finalize the scaling of the fluid flow part, we adopt the kinematic viscosity $\nu$ to quantify the so-called viscous fluxes in the NavierStokes equations. In order to scale the convection-diffusion-reaction equation for the active microorganism concentration $C$ we adopt the same time- and length-scales, and use the average concentration at the inlet of the reactor, $\bar{C}_{0}$, as reference scale for the concentration. The intensity is scaled using the average value of the lamp intensity, $\bar{I}$, as reference scale. Altogether, the parameters with asterisks are scaled as follows

$$
\begin{array}{rlr}
\left(z^{*}, r^{*}\right) & =R_{2}(z, r) & \mathrm{m} \\
\mathbf{v}^{*} & =u_{0} \mathbf{v} & \mathrm{m} / \mathrm{s} \\
t^{*} & =\frac{R_{2}}{u_{0}} t & \mathrm{~s} \\
C^{*} & =\bar{C}_{0} C & 1 / \mathrm{m}^{3} \\
I^{*} & =\bar{I} I & \mathrm{~W} / \mathrm{m}^{2} \\
E^{*} & =\frac{E}{R_{2}} & 1 / \mathrm{m} \\
p^{*} & =\rho u_{0}^{2} p & \mathrm{~kg} / \mathrm{ms}^{2} \\
\alpha & =R_{1} / R_{2} . &
\end{array}
$$

This yields a normalized disinfection reaction rate $K$ that is given by

$$
K=\frac{I}{r} \exp (-E(r-\alpha))=I f(r) .
$$

Here $r$ denotes the scaled radial coordinate, $I$ is the scaled intensity, and the scaled absorbance is denoted by $E$. The susceptibility factor is included in the overall amplitude of the total disinfection rate that results from the combined scaling factors, as we shall see later on.

Written out in full, the scaled model equations for the fluid flow in cylindrical coordinates are given by (see [9] pp. 59-60) the continuity equation:

$$
\frac{1}{r} \frac{\partial}{\partial r}\left(r v_{r}\right)+\frac{1}{r} \frac{\partial v_{\theta}}{\partial \theta}+\frac{\partial v_{z}}{\partial z}=0
$$


and by the conservation of momentum:

$$
\begin{aligned}
& \frac{\partial v_{r}}{\partial t}+v_{r} \frac{\partial v_{r}}{\partial r}+\frac{v_{\theta}}{r} \frac{\partial v_{r}}{\partial \theta}-\frac{v_{\theta}^{2}}{r}+v_{z} \frac{\partial v_{r}}{\partial z} \\
= & -\frac{\partial p}{\partial r}+\frac{1}{\operatorname{Re}}\left[\frac{\partial}{\partial r}\left(\frac{1}{r} \frac{\partial}{\partial r}\left(r v_{r}\right)\right)+\frac{1}{r^{2}} \frac{\partial^{2} v_{r}}{\partial \theta^{2}}-\frac{2}{r^{2}} \frac{\partial v_{\theta}}{\partial \theta}+\frac{\partial^{2} v_{r}}{\partial z^{2}}\right] \\
& \frac{\partial v_{\theta}}{\partial t}+v_{r} \frac{\partial v_{\theta}}{\partial r}+\frac{v_{\theta}}{r} \frac{\partial v_{\theta}}{\partial \theta}+\frac{v_{r} v_{\theta}}{r}+v_{z} \frac{\partial v_{\theta}}{\partial z} \\
= & -\frac{1}{r} \frac{\partial p}{\partial \theta}+\frac{1}{\operatorname{Re}}\left[\frac{\partial}{\partial r}\left(\frac{1}{r} \frac{\partial}{\partial r}\left(r v_{\theta}\right)\right)+\frac{1}{r^{2}} \frac{\partial^{2} v_{\theta}}{\partial \theta^{2}}+\frac{2}{r^{2}} \frac{\partial v_{r}}{\partial \theta}+\frac{\partial^{2} v_{\theta}}{\partial z^{2}}\right] \\
& \frac{\partial v_{z}}{\partial t}+v_{r} \frac{\partial v_{z}}{\partial r}+\frac{v_{\theta}}{r} \frac{\partial v_{z}}{\partial \theta}+v_{z} \frac{\partial v_{z}}{\partial z} \\
= & -\frac{\partial p}{\partial z}+\frac{1}{\operatorname{Re}}\left[\frac{1}{r} \frac{\partial}{\partial r}\left(r \frac{\partial v_{z}}{\partial r}\right)+\frac{1}{r^{2}} \frac{\partial^{2} v_{z}}{\partial \theta^{2}}+\frac{\partial^{2} v_{z}}{\partial z^{2}}\right] .
\end{aligned}
$$

Here $\operatorname{Re}=u_{0} R_{2} / \nu$ is the Reynolds number, which quantifies the ratio of the convective forces over the viscous forces in the flow. We use cylindrical coordinates $(r, \theta, z)$ (Cf. Figure. 1) with velocity components $\left(v_{r}, v_{\theta}, v_{z}\right)$ and dimensionless pressure $p$. The convection-diffusion-reaction equation for the active microorganism concentration can be written as:

$\frac{\partial C}{\partial t}=-\left(v_{r} \frac{\partial C}{\partial r}+v_{\theta} \frac{1}{r} \frac{\partial C}{\partial \theta}+v_{z} \frac{\partial C}{\partial z}\right)+\frac{1}{\mathrm{Pe}}\left[\frac{1}{r} \frac{\partial}{\partial r}\left(r \frac{\partial C}{\partial r}\right)+\frac{1}{r^{2}} \frac{\partial^{2} C}{\partial \theta^{2}}+\frac{\partial^{2} C}{\partial z^{2}}\right]-\operatorname{Da} K C$,

Here, $\mathrm{Pe}=u_{0} R_{2} / D$ is the Peclet number, which, analogous to Re, measures the relative importance of the convective mass transfer over the diffusive mass transfer. $\mathrm{Da}=\varepsilon \bar{I} \alpha R_{2} / u_{0}$ is the Damköhler number, which indicates the chemical reaction rate relative to the convective mass transfer rate. The description of the fluid flow and concentration dynamics given in (8)-(10) does not allow further analytical treatment in its general form. Therefore, we proceed with analyzing the velocity and concentration profiles by introducing a number of simplifying assumptions. Most importantly, these limit the applicability of the model to laminar flow conditions, as apply, e.g., to UV treatment in cider plants [6]. Further, suitable boundary conditions are chosen, together with the controlled and measured variables.

\subsection{Laminar velocity field model}

In addition to the laminar flow assumption, we assume that the the reactor is very long. This implies that the fluid motion is in the $z$ direction only, i.e., $v_{\theta}=v_{r}=0$. This requires that the Reynolds number is sufficiently small and that the flow has become fully developed before the reactor inlet. In other words, there are no entry effects, and as a consequence the velocity profile depends on $r$ and $t$, and not on $z$ nor on $\theta$. As a result we have $\mathbf{v}=\left(0,0, v_{z}(r, t)\right)$, which satisfies (8) and also implies that $\frac{\partial^{2} v_{z}}{\partial \theta^{2}}=\frac{\partial^{2} v_{z}}{\partial r^{2}}=0$. Therefore, the NavierStokes system (9) contains only one non-trivial equation. Only the longitudinal 
momentum equation needs to be retained, yielding

$$
\frac{\partial v_{z}}{\partial t}=-\frac{\partial p}{\partial z}+\frac{1}{\operatorname{Re}}\left[\frac{1}{r} \frac{\partial}{\partial r}\left(r \frac{\partial v_{z}}{\partial r}\right)\right] .
$$

Taking the derivative of this equation with respect to $z$, and using the fact that $v_{z}$ does not depend on $z$, yields that $\partial p / \partial z$ is independent of $z$. Since the pressure does not depend on $r$ or $\theta$, the pressure derivative depends on $t$ only. We set

$$
-\frac{\partial p}{\partial z}(t)=\beta(t)
$$

Further we assume no-slip boundary conditions at the walls, which is the most common choice for incompressible fluid flow,

$$
v_{z}(\alpha)=v_{z}(1)=0 \text {. }
$$

This completes the laminar fluid-mechanics description of the reactor flow.

\subsection{Convection-diffusion-reaction equation}

Since the velocity field, the UV-radiation field, the initial condition, and the geometry are assumed to be independent of $\theta$, the concentration of active microorganisms will also not depend on $\theta$. Hence, (10) becomes

$$
\frac{\partial C}{\partial t}=-v_{z} \frac{\partial C}{\partial z}+\frac{1}{\operatorname{Pe}}\left[\frac{1}{r} \frac{\partial}{\partial r}\left(r \frac{\partial C}{\partial r}\right)+\frac{\partial^{2} C}{\partial z^{2}}\right]-\mathrm{Da} K C .
$$

To complete the formulation for $C$ we introduce boundary conditions in the $z$ and $r$-direction. First, we assume that the concentration at the inlet is wellmixed, making it a function of $t$, but not of $r$. Moreover, we assume that there is no concentration gradient in $z$ at the inlet. This corresponds with the absence of UV radiation for $z \leq 0$. Thus

$$
C(0, r, t)=C_{0}(t), \quad \frac{\partial C}{\partial z}(0, r, t)=0 .
$$

At the walls the velocity is zero and the only mass transport comes from diffusion. The diffusion is small compared to the chemical reaction rate if $1 / \mathrm{DaPe} \ll 1$. We restrict ourselves to such cases and assume that the concentration at the walls is zero, as the radiation will have sufficient time to eliminate the active microorganisms:

$$
C(z, \alpha, t)=0, \quad C(z, 1, t)=0
$$

This completes the formulation for the dynamics of the microorganism concentration. Recapitulating, the basic model describing the flow and concentration 
inside the reactor is

$$
\begin{aligned}
\frac{\partial v_{z}(r, t)}{\partial t}= & \beta(t)+\frac{1}{\operatorname{Re}}\left[\frac{1}{r} \frac{\partial}{\partial r}\left(r \frac{\partial v_{z}(r, t)}{\partial r}\right)\right] \\
v_{z}(\alpha, t)= & v_{z}(1, t)=0 \\
\frac{\partial C(z, r, t)}{\partial t}= & -v_{z}(r, t) \frac{\partial C(z, r, t)}{\partial z}+\frac{1}{\operatorname{Pe}}\left[\frac{1}{r} \frac{\partial}{\partial r}\left(r \frac{\partial C(z, r, t)}{\partial r}\right)+\frac{\partial^{2} C(z, r, t)}{\partial z^{2}}\right] \\
& -\operatorname{Da} K(r, t) C(z, r, t) \\
C(0, r, t)= & C_{0}(t), \frac{\partial C}{\partial z}(0, r, t)=0, \quad C(z, \alpha, t)=C(z, 1, t)=0 .
\end{aligned}
$$

where $v_{z}$ is a function of $r$ and $t$, and $C$ a function of $z, r$ and $t$. In practical UV-treatment, control is exerted with $I(t)$, the intensity of the lamp, see (7). The average concentration at the outflow is a natural monitoring parameter that needs to be controlled. Here, we define the average concentration in terms of the total outflow of microorganisms relative to the average velocity of the liquid. This is defined by

$$
\bar{C}(L, t)=\frac{\int_{\alpha}^{1} C(L, r, t) v_{z}(r) r d r}{\int_{\alpha}^{1} v_{z}(r) r d r} .
$$

The control will be aimed at reducing $\bar{C}(L, t)$ below a pre-set acceptance level, by adjusting the input $I$. We turn to this in the next section.

\subsection{Analytical solutions for special cases}

To get some insight into the disinfection process, the laminar flow and corresponding concentration profiles are analyzed next. For a reactor with a constant flow rate, i.e., $\partial v_{z} / \partial t=0$ and $\beta$ constant, the solution of the differential equation for the velocity in (17) is given by

$$
v_{z}(r)=-\frac{\beta \operatorname{Re}}{4}\left[r^{2}+\frac{1-\alpha^{2}}{\ln (\alpha)} \ln (r)-1\right] .
$$

This shows a characteristic quadratic profile, reminiscent of the Poiseuille profile, with logarithmic corrections arising from the UV lamp along the center of the cylinder. After choosing the parameters from Table 1 for the cider plant in section 2.7, we get $\beta R e=41.5$. The parameter $\alpha$ can be seen as a design parameter, which is for example small for a wide reactor with a thin lamp. Figure 2 shows the velocity profile for different values of $\alpha$. The velocity profile decreases strongly with $\alpha$. The shape of the profile is more or less parabolic as in an ordinary tube flow. The peak shifts a little bit from the center of the radius for lower $\alpha$. Using this velocity profile, we analyze the concentration of active microorganisms next. As mentioned above, we restrict to cases in which diffusive transport is negligible compared to convection and UV irradiation, or equivalently, $\mathrm{Pe} \ll 1$ and $\mathrm{Pe} \gg \mathrm{Da}$. For the particular case of UV disinfection in a cider plant [6], this is the case, and in section 2.6 the influence of mass diffusion 


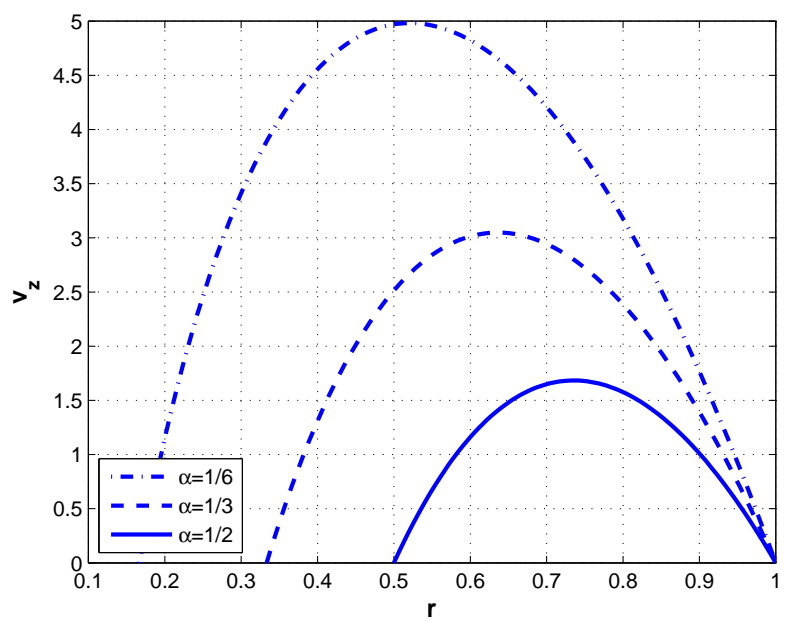

Figure 2: The velocity profile $v_{z}(r)$ for different values of $\alpha$, with $\beta \operatorname{Re}=1.1610^{3}$.

is shown to be negligible by numerical analysis. This motivates neglecting mass diffusion. By doing so, the third equation in (17) becomes

$$
\begin{aligned}
\frac{\partial C}{\partial t} & =-v_{z} \frac{\partial C}{\partial z}-\operatorname{Da} K C \\
C(0, r, t) & =C_{0}(t),
\end{aligned}
$$

which is a standard convection-reaction process. Note that with $v_{z}(r)$ from (20) the boundary conditions in (17) are satisfied. In view of the control design that focuses on the output concentration $C$ at $z=L$ we may readily derive

$$
C(L, r, t)=C_{0}\left(t-\frac{L}{v_{z}(r)}\right) \exp \left(-\mathrm{Da} \int_{t-\frac{L}{v_{z}(r)}}^{t} K(r, \tau) d \tau\right) .
$$

This expresses the instantaneous solution $C(L, r, t)$ in terms of the inflow-value at a previous time $t-t_{r}(r)$ where the residence time $t_{r}=L / v_{z}(r)$. In addition, the accumulated effect of the UV irradiation at distance $r$, acquired during a time-interval $t_{r}$ is expressed by the exponential. To simplify the analysis, assume that $C_{0}$ and $K$ do not depend on $t$. Expression (22) then becomes

$$
C(L, r)=C_{0} \exp \left(-\frac{\mathrm{Da} K(r) L}{v_{z}(r)}\right)
$$

This is plotted on a logarithmic scale for different values of $\alpha$ in Figure 3 . The physical parameters are are the same as in the nominal model that will be introduced in section 2.7. We observe that close to the boundaries $r=\alpha$ and $r=1$ the concentration drops strongly - this is associated with the very 
long residence time of microorganisms that enter the reactor close to a wall. Conversely, only the 'inner' region of the reactor contributes significantly to the outflow of still active microorganisms. Hence, especially for quite large values of $\alpha$, the contribution of $C(L, r)$ to $\bar{C}(L)$ is significant only in a small part of the reactor. Expressions (19) and (23) illustrate that $\bar{C}(L)$ is influenced strongly by the smallest residence time $L / v_{z}$.

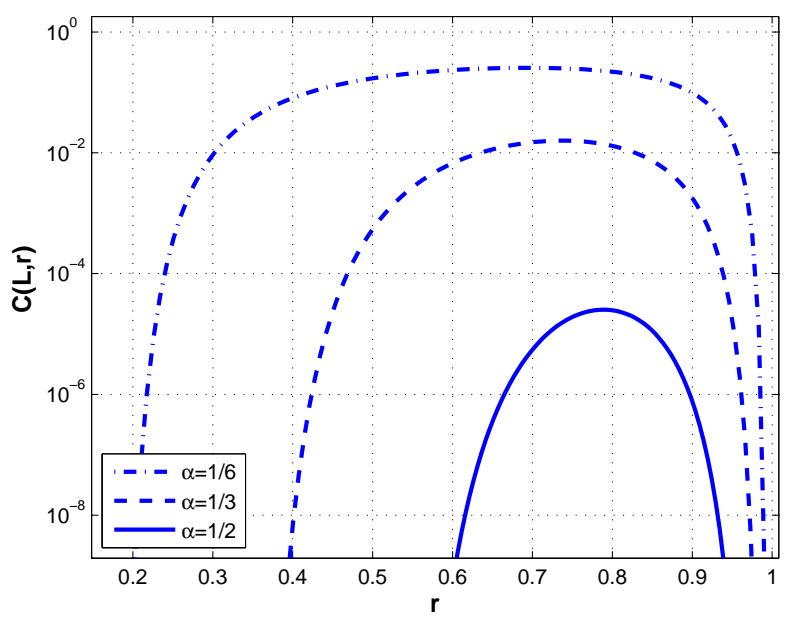

Figure 3: Concentration profile of $C(L, r)$ for different values of $\alpha$. The relevant parameter values are listed in Table 1.

\subsection{Numerical analysis of the basic model}

In this section the influence of mass diffusion is investigated numerically for the steady state form of the basic model (17). For that, we define a discrete update scheme and consequently we determine an appropriate grid. The parameter values that are used are the same as in the nominal model of section 2.7 , and they are listed in Table 1 . The discrete update scheme for the concentration 
plus boundary conditions is

$$
\begin{aligned}
v_{j} \frac{C_{j, k+1}-C_{j, k}}{\Delta z}= & \frac{1}{\operatorname{Pe} \Delta r^{2} r_{j}}\left(\left(r_{j}+\frac{1}{2} \Delta r\right) C_{j+1, k}-2 r_{j} C_{j, k}\right. \\
& \left.+\left(r_{j}-\frac{1}{2} \Delta r\right) C_{j-1, k}\right) \\
& +\frac{1}{\operatorname{Pe} \Delta z^{2}}\left(C_{j, k+1}-2 C_{j, k}+C_{j, k-1}\right)-\mathrm{Da} K_{j} C_{j, k} \\
C_{n_{r}, k}= & C_{0, k}=0 \\
C_{2 . . n_{r}-1,-1}= & C_{2 . . n_{r}-1,0}=C_{0} \\
\bar{C}_{n_{z}}= & \frac{\sum_{j=1}^{n_{r}-1}\left(C_{n_{z}, j} v_{j} r_{j}+C_{n_{z}, j+1} v_{j+1} r_{j+1}\right) \Delta r}{\sum_{j=1}^{n_{r}-1}\left(v_{j} r_{j}+v_{j+1} r_{j+1}\right) \Delta r},
\end{aligned}
$$

with $r_{j}=j \Delta r$. Here, $C_{j, k}=C(j \Delta r, k \Delta z)$. The subscript $j=1 \ldots n_{r}$ denotes the discrete radial space with mesh-size $\Delta r$, and $k=1 \ldots n_{z}$ denotes the discrete longitudinal space with mesh-size $\Delta z$. A central discretisation in $r$ and $z$ is used, except for the convective term, which has an upwind discretisation. For the initial condition an Euler step is used. Further, $C=C_{0}$ at the inlet, except for the walls, where $C \equiv 0 . \bar{C}(L)$ is computed with the trapezoidal integration rule. The velocity profile is taken from the model in (17). The left plot in Figure 4 shows $\bar{C}(L)$ as a function of the number of grid points in $z$-direction, $n_{z}$, with a fixed value of $n_{r}=15$. A sufficient convergence is attained for $n_{z}=10^{4}$, since for higher values of $n_{z}$ the average concentration is more or less the same. The right plot in Figure 4 shows $\bar{C}(L)$ as a function of the number of grid points in $r$ direction, $n_{r}$, with a fixed value of $n_{z}=10^{4}$. A sufficient convergence is attained for $n_{r}=15$. Altogether, a sufficient fine grid consists of $n_{z} \times n_{r}=10^{4} \times 15$ grid points, and this is the grid that will be used from now on. The resolution of the grid in $z$-direction is four orders higher than in $r$-direction. This is partially explained as follows. The convective term is discretised as

$$
v_{j} \frac{\partial C_{j, k}}{\partial z}=v_{j} \frac{C_{j, k+1}-C_{j, k}}{\Delta z}+v_{j} \frac{\Delta z}{2} \frac{\partial^{2} C_{j, k}}{\partial z^{2}}+\mathcal{O}\left(\Delta z^{2}\right),
$$

where the second r.h.s. term is the discretisation error of order $\Delta z$. The solution of $C$ without mass diffusion is given by equation (23). Inserting its second derivative gives an error of

$$
\frac{\Delta z}{2 v_{j}}\left(\mathrm{Da} K_{j}\right)^{2} C_{0} \exp \left(-\frac{\mathrm{Da} K_{j} z}{v_{j}}\right)
$$

which grows infinitely large for $(z, r) \rightarrow(0, \alpha)$ and $(z, r) \rightarrow(0,1)$, since $v=0$ at the walls. However, it was shown in Figure 3 that the concentration near the wall has a very small influence on the average concentration. For the bulk of the flow, the error is maximal in $z=0$, and in the order of $\Delta z 10^{2}$, which means that the grid is sufficient fine if $\Delta z \ll 10^{-2}$, and since $n_{z} \Delta z=L$, this 

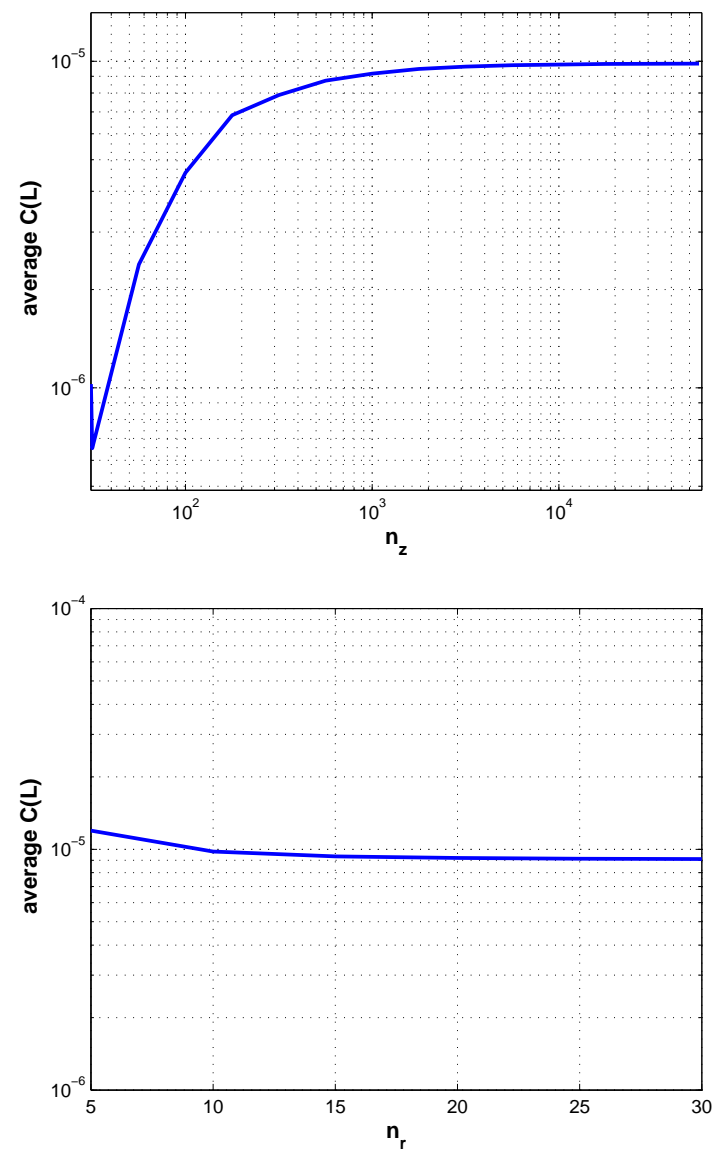

Figure 4: The numerical solution of $\bar{C}(L)$ as a function of $n_{z}$ (left) with $n_{r}=15$, and as a function of $n_{r}$ (right) with $n_{z}=10^{4}$.

means that $n_{z} \gg 10^{2}$. The discretisation error in radial direction is caused by the discretisation of the diffusive term, which has an error of

$$
\frac{1}{\mathrm{Pe}} \frac{\Delta r^{2}}{6} \frac{\partial^{3} C_{j, k}}{\partial r^{3}}
$$

Since this decreases quadratically with $\Delta r$, and because $\frac{1}{\mathrm{Pe}}$ is very small, this gives an indication on why a coarse grid in $r$-direction is sufficient.

Figure 5 shows a contour plot of $C$ for a stationary flow in the domain $r \in[\alpha, 1]$, and $z \in[0, L]$. The lamp is placed on the bottom horizontal axis. The relatively large distances between the contour lines indicate a small concentration gradient in the center, caused by the high convection. As a contrast, the concentration gradient is very steep near the walls at the inlet. 
We will now investigate the influence of mass diffusion. The significance

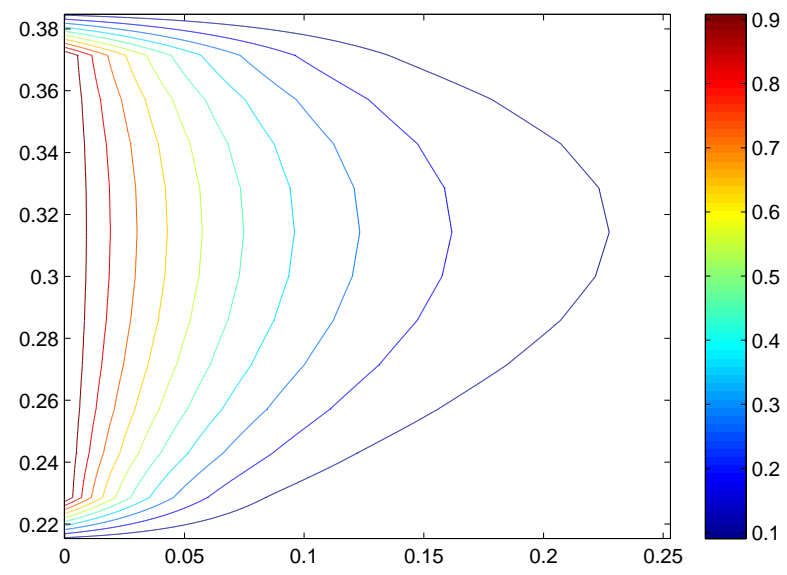

Figure 5: Contourplot of $C(z, r)$ for the apple cider reactor. The horizontal axis denotes the $z$-direction, and the vertical axis the $r$-direction.

of mass diffusion depends on the relative contributions of reaction and mass diffusion, indicated by Da and Pe. We define $\bar{C}(L)_{d}$ and $\bar{C}(L)$ as the average concentrations at $L$ with and without mass diffusion, respectively. Figure 6 shows $\frac{\bar{C}(L)_{d}-\bar{C}(L)}{\bar{C}(L)}$ as a function of Pe and Da. In our case, $\mathrm{Pe}=3.610^{5}$ and $\mathrm{Da}=5.6$, and the difference is smaller than $1 \%$. In addition to the arguments in section 2.5, this motivates the choice of discarding the term $\frac{1}{\operatorname{Pe}}\left[\frac{1}{r} \frac{\partial}{\partial r}\left(r \frac{\partial C(z, r, t)}{\partial r}\right)+\right.$ $\left.\frac{\partial^{2} C(z, r, t)}{\partial z^{2}}\right]$ from (17) in the following.

\subsection{The nominal model}

Using the results of the previous sections, we obtain a model for an apple cider plant. The dimensionless constants for this plant are

$$
\mathrm{Pe}=3.510^{5}, \mathrm{Da}=5.8, \mathrm{Re}=1.310^{3}, \text { and } \alpha=0.5,
$$

and they are determined by using parameter values specific for apple cider, [6]. In Table 1 all the physical constants are listed ${ }^{1}$. From section 2.1 we have that $\mathrm{Da}=\varepsilon \bar{I} \alpha R_{2} / u_{0}$, where $\bar{I}$ is the average lamp intensity. The light intensity is determined such that it gives a ' 5 log reduction' of the inlet concentration of the Escherichia coli 0157:H7 bacteria, i.e., $\bar{C}(L)=10^{-5} C_{0}$. Furthermore, we assume that the flow rate through the reactor is constant. By section 2.5 this

\footnotetext{
${ }^{1}$ The viscosity $\eta$ is a factor 10 higher than that of water. However, this is the value that is mentioned several times in [6], so we assume this to be a realistic value.
} 


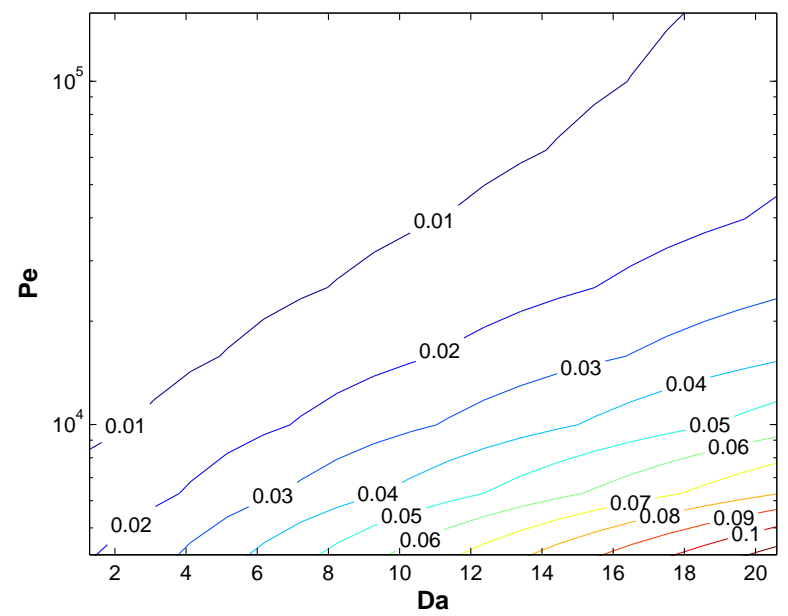

Figure 6: Isolines of the percentage difference $\frac{\bar{C}(L)_{d}-\bar{C}(L)}{\bar{C}(L)}$ for different values of Pe and Da.

\begin{tabular}{|cc|cc|}
\hline$R_{1}$ & $0.2 \mathrm{~m}$ & $R_{2}$ & $0.4 \mathrm{~m}$ \\
$E^{*}$ & $1.1 \mathrm{~m}^{-1}$ & $\epsilon$ & $0.2624 \mathrm{~m}^{2} \mathrm{~J}^{-1}$ \\
$\frac{d p^{*}}{d z^{*}}$ & $-0.9 \mathrm{kgm}^{-2} \mathrm{~s}^{-2}$ & $\eta$ & $310^{-2} \mathrm{kgm}^{-1} \mathrm{~s}^{-1}$ \\
$I^{*}$ & $10.5 \mathrm{Wm}^{-2}$ & $C_{0}^{*}$ & $10^{7} \mathrm{~m}^{-3}$ \\
$C_{p}$ & $5.1110^{3} \mathrm{Jkg}^{-1} \mathrm{~K}^{-1}$ & $\lambda$ & $0.55 \mathrm{Wm}^{-1} \mathrm{~K}^{-1}$ \\
$L^{*}$ & $1 \mathrm{~m}$ & $u_{0}$ & $9.410^{-2} \mathrm{~ms}^{-1}$ \\
\hline
\end{tabular}

Table 1: Physical parameters of the apple cider plant.

implies that $v_{z}$ is given by (20). Combining (7), (20) and (21), we obtain the following nominal model

$$
\begin{aligned}
v_{z}(r) & =-\frac{\beta \mathrm{Re}}{4}\left[r^{2}+\frac{1-\alpha^{2}}{\ln (\alpha)} \ln (r)-1\right] \\
\frac{\partial C(z, r, t)}{\partial t} & =-v_{z}(r) \frac{\partial C(z, r, t)}{\partial z}-\operatorname{Da} I(t) f(r) C(z, r, t) \\
C(0, r, t) & =C_{0}(t) .
\end{aligned}
$$

In this model, we regard $I(t)$ as the control input, and $C_{0}(t)$ as the disturbance. The measurement is given by (19).

\section{Model reduction for the nominal model}

In the nominal model (29) we see that our control input $I(t)$ gets multiplied with the state $C(z, r, t)$, and so it is a non-linear model. Furthermore, it is 
a distributed parameter model, which is harder for control design. Using linearization and reduction via balancing, we will obtain a first order linear model for which the control design is easy.

Since the nominal model is given in the scaled variables, we take as reference values $C_{0}^{r e f}=1, I^{r e f}=1$. For these reference values, the solution of (29) gives the reference concentration profile

$$
C^{r e f}(z, r)=C_{0}^{r e f} \exp \left(-\frac{\operatorname{Da} f(r) z}{v_{z}(r)}\right) .
$$

Defining $I(t)=I^{\text {ref }}+I^{\text {var }}(t), C_{0}(t)=C_{0}^{r e f}+C_{0}^{\text {var }}$, and $C(z, r, t)=C^{\text {ref }}(z, r)+$ $C^{v a r}(z, r, t)$, and linearizing (29) around the reference concentration, gives the following p.d.e. for $C^{v a r}$

$$
\begin{aligned}
\frac{\partial C^{v a r}(z, r, t)}{\partial t}= & -v_{z}(r) \frac{\partial C^{\text {var }}(z, r, t)}{\partial z} \\
& -\operatorname{Da} I^{\text {var }}(t) f(r) C^{r e f}(z, r)-\operatorname{Da} f(r) C^{v a r}(z, r, t) \\
C^{v a r}(0, r, t)= & C_{0}^{\text {var }}(t)
\end{aligned}
$$

We use this to obtain the transfer functions from input and disturbances to the state. Let $\widehat{x}$ denote the Laplace transform of the variable $x$. Then after Laplace transform with respect to time, equation (31) becomes

$$
\begin{aligned}
s \widehat{C}^{v a r}(z, r, s)= & -v_{z}(r) \frac{\partial \widehat{C}^{v a r}(z, r, s)}{\partial z} \\
& -\operatorname{Da} \widehat{I}^{\text {var }}(s) f(r) C^{r e f}(z, r)-\operatorname{Da} f(r) \widehat{C}^{v a r}(z, r, s) \\
\widehat{C}^{v a r}(0, r, s)= & \widehat{C}_{0}^{\text {var }}(s) .
\end{aligned}
$$

Since the output depends on the concentration at $z=L$, see (19), we solve this p.d.e. for $z=L$. The solution is given by

$$
\begin{aligned}
\widehat{C}^{v a r}(L, r, s)= & \frac{\operatorname{Da} f(r)}{s}\left[\exp \left(-\frac{\operatorname{Da} f(r) L}{v_{z}(r)}\right)-\exp \left(-\frac{s+\operatorname{Da} f(r)}{v_{z}(r)} L\right)\right] \widehat{I}^{\text {var }}(s) \\
& +\exp \left(-\frac{s+\operatorname{Da} f(r)}{v_{z}(r)} L\right) \widehat{C}_{0}^{\text {var }}(s) \\
= & \tilde{G}_{1}(r, s) \widehat{I}^{\text {var }}(s)+\tilde{G}_{2}(r, s) \widehat{C}_{0}^{\text {var }}(s) .
\end{aligned}
$$

The transfer function $\tilde{G}_{1}(r, s)$ is a non-rational function in $s$. Hence it is not straightforward to find a balanced realization of it. Therefore, we approximate $\tilde{G}_{1}(r, s)$ by a rational one. For fixed $r$ we use the first order Padé approximation, i.e.,

$$
\begin{aligned}
\tilde{G}_{1}(r, s) & \approx \frac{\tilde{G}_{1}(r, 0)^{2}}{-\frac{\partial \tilde{G}_{1}(r, 0)}{\partial s} s+\tilde{G}_{1}(r, 0)} \\
& =\frac{\operatorname{Da} f(r) C^{r e f}(L, r)}{s+\frac{2 v_{z}(r)}{L}} .
\end{aligned}
$$


For the transfer function from $I^{v a r}(t)$ to $\overline{C^{v a r}}(L, t)$ we obtain the following approximation, see (19)

$$
\bar{G}_{1}(r, s)=\frac{\int_{\alpha}^{1} \frac{\operatorname{Da} f(r) C^{r e f}(L, r)}{s+\frac{2 v_{z}(r)}{L}} v_{z}(r) r d r}{\int_{\alpha}^{1} v_{z}(r) r d r} .
$$

A state space realization of this is given by

$$
\begin{aligned}
\frac{d x(r, t)}{d t} & =a(r) x(r, t)+b(r) I^{v a r}(t) \\
\overline{C^{v a r}}(L, t) & =\frac{1}{\overline{v r}} \int_{\alpha}^{1} x(r, t) v_{z}(r) r d r,
\end{aligned}
$$

where

$$
a(r)=-2 \frac{v_{z}(r)}{L}, \quad b(r)=\operatorname{Da} f(r) C^{r e f}(L, r) \text { and } \overline{v r}=\int_{\alpha}^{1} v_{z}(r) r d r .
$$

Since the state $x$ at time $t$ is a function of $r$, this is an infinite-dimensional system. Next, we approximate this by a finite-dimensional one. Therefore we discretize $x(r, t)$ with respect to $r$. The new state vector is $x(t)=\left[x\left(r_{1}, t\right) \ldots x\left(r_{n}, t\right)\right]^{T}$, and the model becomes

$$
\begin{aligned}
\frac{d x}{d t}(t) & =\mathbf{A} x(t)+\mathbf{B} I^{v a r}(t) \\
\overline{C^{v a r}}(L, t) & =\mathbf{C} x(t)
\end{aligned}
$$

with

$$
\begin{aligned}
\mathbf{A} & =\operatorname{diag}\left[a\left(r_{1}\right), \ldots, a\left(r_{n}\right)\right] \\
\mathbf{B} & =\left[b\left(r_{1}\right), \ldots, b\left(r_{n}\right)\right]^{T} \\
\mathbf{C} & =\frac{1}{\overline{v r}}\left[\frac{v\left(r_{1}\right) r_{1}}{2}, v\left(r_{2}\right) r_{2}, \ldots, v\left(r_{n-1}\right) r_{n-1}, \frac{v\left(r_{n}\right) r_{n}}{2}\right] .
\end{aligned}
$$

Matrix $\mathbf{C}$ is obtained by trapezoidal integration. Next, a low order approximation of this system is made, using input/output balanced truncation. To get an idea of the suitable order down to which the system can be truncated, the Hankel singular values of system (38) are computed. These values give an indication of the dominance of the states of the input/ouput balanced system in terms of energy, and are determined by solving the Riccati equations for $P$ and $Q$.

$$
\begin{aligned}
\mathbf{A} P+P \mathbf{A}^{T} & =-\mathbf{B B}^{T} \\
\mathbf{A}^{T} Q+Q \mathbf{A} & =-\mathbf{C}^{T} \mathbf{C} .
\end{aligned}
$$

The Hankel singular values are defined as the eigenvalues of the product $P Q$ :

$$
\sigma_{H}=\sqrt{\lambda_{i}(P Q)}
$$




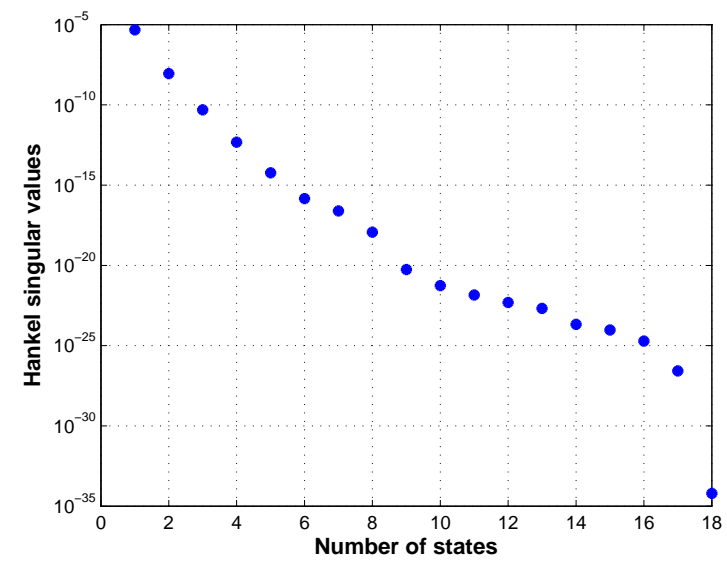

Figure 7: The Hankel singular values of the laminar flow system without diffusion.

Figure 7 shows the Hankel singular values of system (38). Since the first Hankel value is three decades larger than the next one, it is clear that the first state of the balanced system is absolutely dominant, and the rest of the states is negligible, and thus that the dynamics from $I(t)$ to $\bar{C}(L, t)$ are mainly of first order. The balancing is done by a state transformation $x_{b}=x^{T}$, where $x_{b}$ is the state vector of the balanced system. The first state of the balanced system, $\left[\begin{array}{llll}1 & 0 & \ldots & 0\end{array}\right]^{T}$, is the state that corresponds to the first Hankel singular value. The states of the balanced system have no physical interpretation. For physical interpretation, $x_{b}$ is transformed back to $x(r)=T^{-1} x_{b}$. The elements in the vector $x_{b}$ indicate how the different parts of the flow in $r$ contribute to the dominant state $x$, and thus how they contribute to the dynamics in $\bar{C}(L, t)$. Figure 8 shows $-x(r)$ and $C(L, r)$, and it is clear that the contribution of $r$ to $\bar{C}(L, t)$ corresponds to the height of $C(L, r)$, meaning that the dynamics at $r$ for which $C(L, r, t)$ is maximal dominates the dynamics of $\bar{C}(L, t)$.

The balanced system is truncated down to a first order system, with $\left(A_{\text {red }}, B_{\text {red }}\right)$ the truncated system matrices (see for example [24] for details).

$$
\frac{d \overline{C^{v a r}}(L, t)}{d t}=A_{r e d} \overline{C^{v a r}}(L, t)+B_{r e d} I^{v a r}(t) .
$$

Using the constants of our nominal model, we have $A_{\text {red }}=-1.2, B_{\text {red }}=$ $-7.310^{-3}$.

To see whether all approximations have not discarded any essential dynamics, the Bode plot of (41) is compared to that of $\tilde{G}_{1}(s)$ in (33) with (19), see Figure 9. It is clear that (41) is a somewhat crude approximation of (33) with output (19), which nevertheless captures the essential dynamics of the model, namely the static gain and the time constant. Hence, we use (41) as our model for controller design. Using (33), (19), and (41) we have the following model in the 


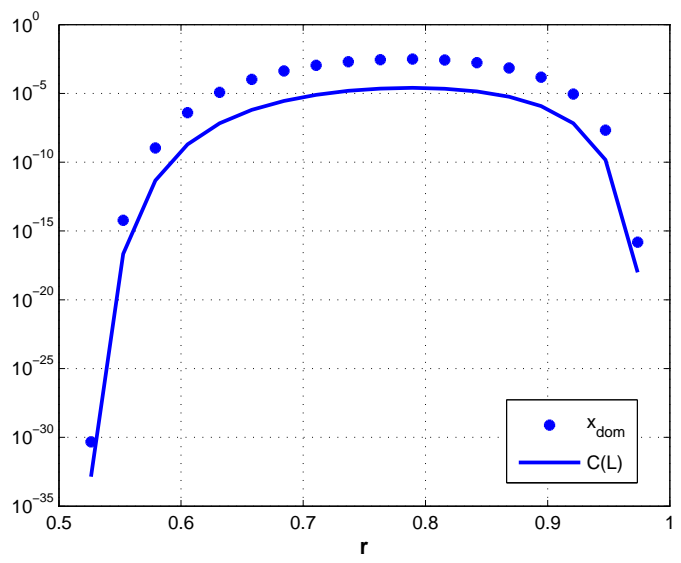

Figure 8: Contributions of the discrete parts of $r$ to the dominant system state, and $C(L, r)$.

$s$-domain

$$
\begin{aligned}
\overline{C^{v a r}}(L, s) & =\frac{B_{r e d}}{s-A_{r e d}} \widehat{I}^{v a r}(s)+\frac{1}{\overline{v r}} \int_{\alpha}^{1} \tilde{G}_{2}(r, s) v_{z}(r) r d r \widehat{C}_{0}^{v a r}(s) \\
& :=G_{1}(s) \widehat{I}^{\text {var }}(s)+\tilde{G}_{2}(s) \widehat{C}_{0}^{v a r}(s) .
\end{aligned}
$$

For numerical simulations in the time domain, $\tilde{G}_{2}(s)$ has to be approximated by a rational transfer function. The approximation of $\tilde{G}_{2}(s)$ is made as follows. The amplitude Bode plot of $\tilde{G}_{2}(s)$ is flat. Hence we approximate it by a pure time delay,

$$
\tilde{G}_{2}(s) \approx G_{2}(s)=c \exp (-\tau s) .
$$

with $c=\tilde{G}_{2}(0)$, and $\tau=-\frac{1}{c} \frac{d \tilde{G}_{2}}{d s}(0)$. Figure 10 shows the phase and gain plots of $\tilde{G}_{2}(s)$ and $G_{2}(s)$. The gain as well as the phase of $G_{2}(s)$ matches that $\tilde{G}_{2}(s)$ indistinguishably.

\section{Controller design}

For the approximate model in the $s$-domain (see (42) and (43))

$$
\overline{C^{v a r}}(L, s)=G_{1}(s) \widehat{I}^{v a r}(s)+\tilde{G}_{2}(s) \widehat{C}_{0}^{v a r}(s)
$$

we design a controller. This controller will be tested on our nominal model (29) with output equation (19).

Since no dynamic properties of $C_{0}^{v a r}(t)$ are known, and since $G_{2}(s)$ is a pure time delay, we regard the term $G_{2}(s) \widehat{C}_{0}^{\text {var }}(s)$ as delayed white noise. Since the dynamics of $I^{v a r}$ to $\overline{C^{v a r}}$ is of first order, we design a simple PI controller. The 

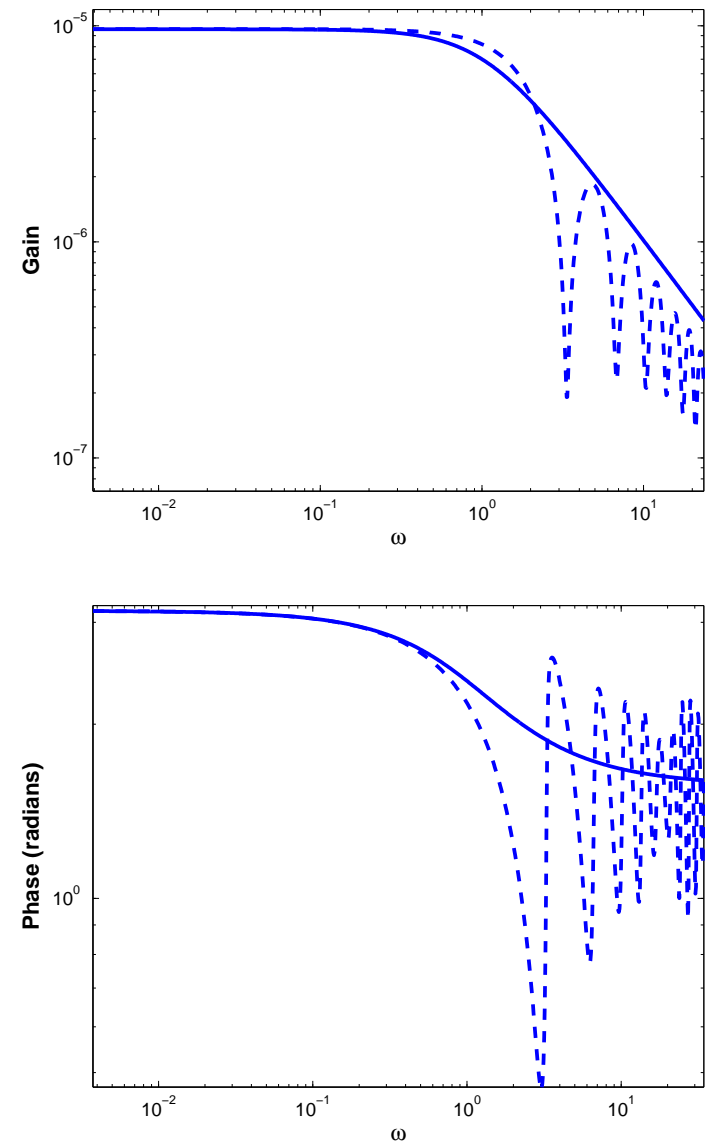

Figure 9: Gain and phase (in radians) plot of $G_{1}(s)$ in model (33) (dashed line), and of model (41) (solid line). Here, $s=i \omega$.

controller should meet the following (standard) design specifications (see also [11])

- Up to some (later specified) crossover frequency, the sensitivity function $S(s)$, should be small, in order to attenuate disturbances, get good command response, and have robustness at low frequencies.

- As a consequence, $T(s)$ should be close to one for low frequencies, and $S(s)$ should be close to one for high frequencies. Here, $S$ is defined as $\frac{1}{1+L}$, with $L=G_{1} K$, and $T=\frac{L}{1+L}$.

Input disturbances with a higher frequency than the crossover frequency are already attenuated by $G_{1}$. Therefore, the ideal crossover frequency of $S$ and $T$ 

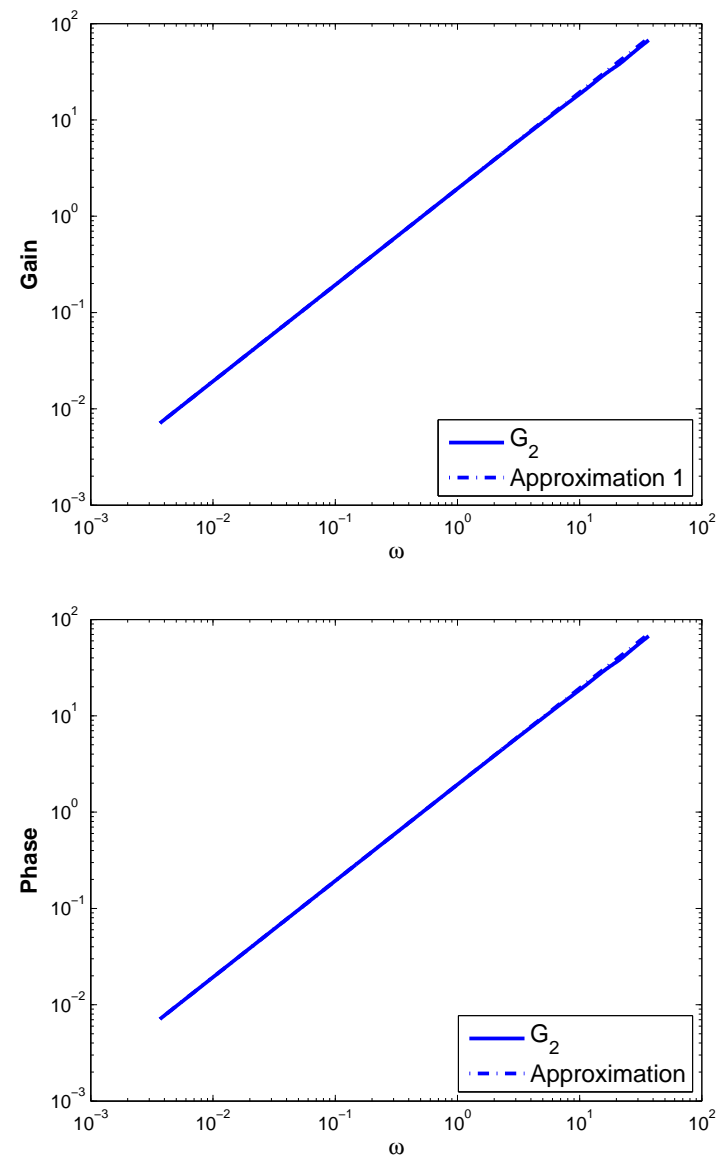

Figure 10: The gain plots (left) and the phase plots (right) of the approximation and the original transfer function.

equals the crossover frequency of $G_{1}:-A_{\text {red }}$. In other words, we have to find a controller $K$ such that $T=\frac{1}{1+\tilde{s}}$, with $\tilde{s}=\frac{s}{-A_{\text {red }}}$. Solving

$$
T=\frac{L}{1+L}=\frac{1}{1+\tilde{s}},
$$

with $L=K G_{1}$, gives the PI controller

$$
K(s)=\frac{k_{1}+s}{k_{2} s},
$$

with $k_{1}=-A_{\text {red }}$ and $k_{2}=\frac{-B_{\text {red }}}{A_{\text {red }}}$. Figure 11 shows the control loop schematically. 


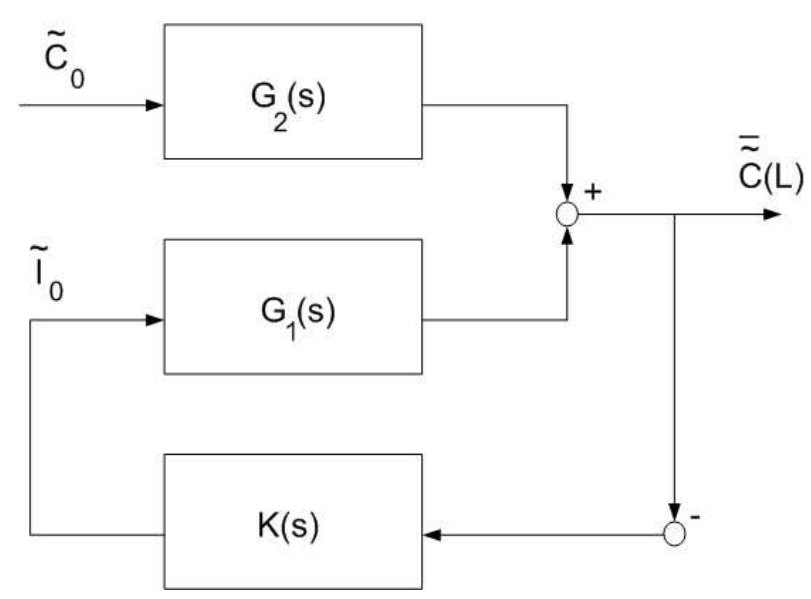

Figure 11: Schematic representation of the controlled model.

\subsection{Simulations}

Simulation studies are conducted to see whether the reduced model (41) is a sufficiently accurate approximation of the nominal model (29). This is done by connecting the controller to both models. The nominal model is solved numerically by a forward Euler method in time, and an upwind scheme in space. This gives the update scheme

$$
\frac{C_{k, j}^{n+1}-C_{k, j}^{n}}{\Delta t}=-v_{j} \frac{C_{k, j}^{n}-C_{k-1, j}^{n}}{\Delta z}-C_{k, j}^{n} \mathrm{Da} I^{n} f_{j} .
$$

Here $v$ is solved analytically in equation (20). Similarly, the update scheme for the controller (46) is, with the reference mean concentration $\bar{C}(L)^{\text {ref }}$ defined as in (19), with $C=C^{r e f}$,

$$
\begin{aligned}
\tilde{I}_{0}^{n+1} & =-\frac{d^{n+1}}{k_{2}}-\frac{1}{k_{2}}\left(\bar{C}(L)^{n}-\bar{C}(L)^{r e f}\right) \\
\frac{d^{n+1}-d^{n}}{\Delta t} & =k_{1}^{n}\left(\bar{C}(L)^{n}-\bar{C}(L)^{r e f}\right) .
\end{aligned}
$$

Here, $n, j$ and $k$ denote the discrete time, radial space, and longitudinal space respectively. The reduced model is a first order o.d.e., and this is simulated within the Matlab Simulink environment. To visualize the difference in output dynamics clearly, $C_{0}(t)$ is disturbed by $A \sin (F t)$, with an amplitude of $A=0.5$ and a frequency of $F=0.8$. For this frequency, the error in gain in Figure 9 is relatively large. The first plot in Figure 12 shows the concentration $\bar{C}(L, t)$ for the reduced model (41) and the nominal model (29). Initially, the difference between the two models is large. This is caused by the transient behavior of the nominal model. The small initial concentration near the inlet is decreased further by the lamp, resulting in a smaller and smaller $\bar{C}(L)$. After the transient 

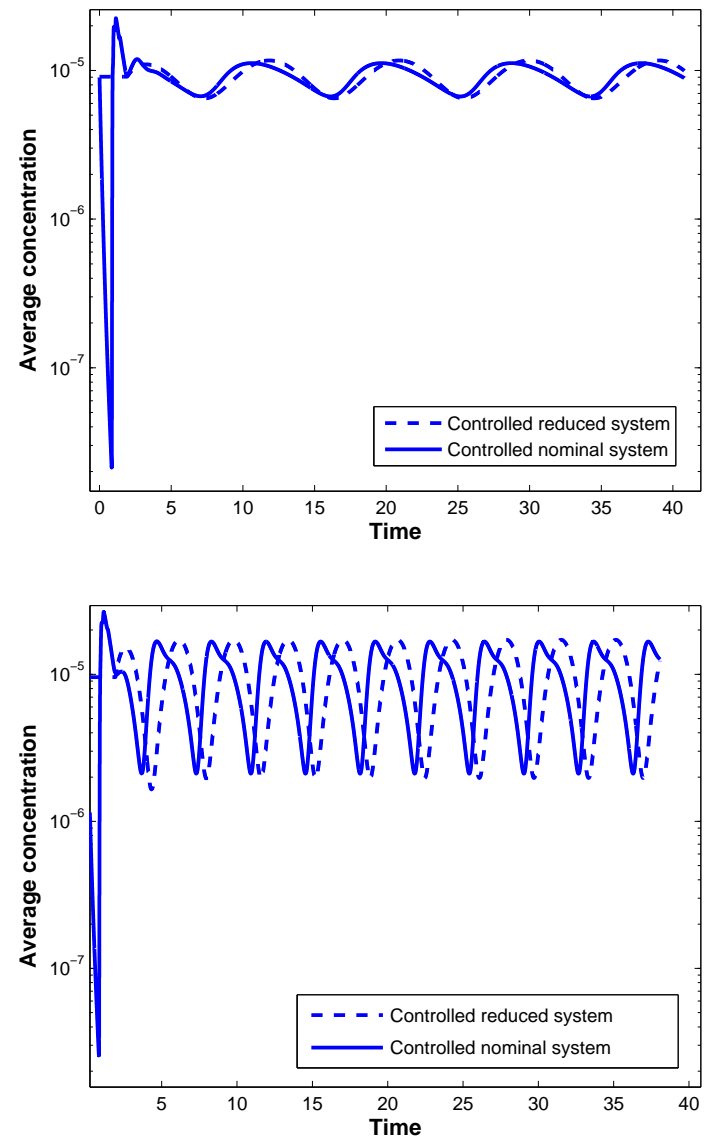

Figure 12: The dynamics of the average concentration $\bar{C}(L, t)$ and the reduced order linearized model (41), and the nonlinear nominal model (29). Left: $A=$ 0.5 and $F=0.8$. Right: $A=0.9$ and $F=2$.

dynamics has gone, the outputs of the nominal and the reduced model match very well. To illustrate the negative influence of large errors in linearization and in the Padé approximation, the amplitude and the frequency of the input disturbance are increased to 0.9 and 2 respectively. The dynamics is shown in the second plot in Figure 12. The higher amplitude causes a larger approximation error in equation (31), and the high frequency causes a larger phase error in Figure 9. Under these circumstances there appears a phase shift between the two outputs. Nevertheless, the controller loses only little performance due to the approximation errors. 


\section{Conclusions}

A basic model was developed, describing the fluid and concentrations dynamics inside an annular disinfection plant. By physical arguments and standard model reduction techniques from system theory it was shown that for a nominal model representing an apple cider plant, a simple classical model-based controller with excellent properties can be designed.

The simulations indicate that for control purposes the reduction process did not discard essential dynamics. This, in turns, indicates that the dynamics of the nominal model are mainly of first order, and that this dynamics can be retrieved by employing standard model reduction techniques.

Up to the balancing, the formulas are analytical and contain all the physical properties of the nominal model. This allows a clear analysis, like in section 2.5. For more complex models, for example with an irregular geometry, or without discarding mass diffusion as was done here, the reduction steps as well as the model simulations can become more numerically involved, since an analytical linearization may not be possible. These practical drawbacks leave the door open for alternative design methods, such as nonlinear control. The next fundamental steps would be to validate the basic model experimentally, and to check the controller performance in a real-life situation.

\section{References}

[1] Anonymous. St. petersburg upgrades water treatment plants with uv systems: The worlds largest uv drinking water disinfection installation began operating in september. Water and wastewater international, 20(7):38, 2005.

[2] H.W. Bode. Network analysis and feedback amplifier design. The Bell telephone laboratories series. Van Nostrand, New York, 1945.

[3] J.R. Bolton. Calculation of ultraviolet fluence rate distributions in an annular reactor: significance of refraction and reflection. Water research, 34:3315-3324, 2000.

[4] A.E. Cassano, C.A. Martin, R.J. Brandi, and O.M. Alfano. Photoreactor analysis and design: Fundamentals and applications. Industrial and engineering chemistry research, 34:2155-2201, 1995.

[5] H. Chmiel, M. Kaschek, C. Blcher, M. Noronha, and V. Mavrov. Concepts for the treatment of spent process water in the food and beverage industries. Desalination, 152(1-3):307-314, 2003.

[6] D.W. Donahue, N. Canitez, and A.A. Bushway. Uv inactivation of e. coli 0157:h7 in apple cider: quality, sensory and shelf life analysis. Journal of food processing and preservation, 28:368-387, 2004. 
[7] W.J. Kowalski et al. Mathematical modeling of ultraviolet germicidal irradiation for air disinfection. Quantitative microbiology, 2:249-270, 2000.

[8] M.M. Hossain, G.B. Raupp, S.O. Hay, and T.N. Obee. Three-dimensional developing flow model for photocatalytic monolith reactors. AIChE Journal, 45(6):1309-1321, June 1999.

[9] L.P.B.M. Janssen and M.M.C.G. Warmoeskerken. Transport phenomena data companion. Delftse Universitaire Pers, 1987.

[10] K.J. Keesman, D. Vries, S. van Mourik, and H. Zwart. Modeling and control of water disinfection process in annular photoreactors. Kos, Greece, July 2007. European control conference.

[11] H. Kwakernaak. Trends in control - a European perspective, chapter Symmetries in control design, pages 17-51. Springer, Heidelberg, 1995.

[12] L. Liberti, M. Notarnicola, G. Boghetich, and A. Lopez. Advanced treatment for municipal wastewater reuse in agriculture. uv disinfection: bacteria inactivation. Journal of water supply: research and technology: aqua, 50(5):275-286, 2001.

[13] L. Liberti, M. Notarnicola, and D. Petruzelli. Advanced treatment for municipal wastewater reuse in agriculture. uv disinfection: parasite removal and by-product formation. Desalination, 152(1-3):315-324, 2003.

[14] P.F.M.M. Roelofs, P.J.J.M. Nooijen, and P.C. Vesseur. Feasability of disinfection of air by UV-radiation for pig houses. Rosmalen: Proefstation voor de varkenshouderij, 1999.

[15] W.T. Runia and S. Boonstra. Disinfection of pythium-infested recirculation water by uv-oxidation technology. 53rd international symposium on crop protection, Gent, 2001.

[16] W.T. Runia and S. Boonstra. Efficacy of uv-oxidation technology against tomato mosaic virus in recirculation water. pages 330-336. 2eme conference internationale sur les moyens alternatifs de lutte contre les organismes nuisibles aux vegetaux, Lille, 2002.

[17] B.F. Severin, M.T. Suidan, B.E. Rittmann, and R.S. Engelbrecht. Inactivation kinetics in a flowthrough uv reactor. Journal of the water pollution control federation, 56:164-169, 1984.

[18] M.T. Suidan and B.F. Severin. Light intensity models for annular uv disinfection reactors. AIChE journal, 32(11):1902-1909, 1986.

[19] M.V. Veazey. Treatment system combines uv disinfection with uvoxidation. Materials performance, 43(12):46, December 2004. 
[20] R.W. Ward and G.M. DeGrave. Residual toxicity of several disinfectants in domestic wastewater. Journal of water pollution control federation, 50(1):46-60, 1978.

[21] I. Woitenko, M.K. Stinson, and R. field. Challenges of combined sewer overflow disinfection by ultraviolet light irradiation. Critical reviews in environmental science and technology, 31(3):223-239, 2000.

[22] H.B. Wright and G. Sakamoto. Uv dose required to achieve incremental $\log$ inactivation of bacteria, viruses and protozoa. Technical report, Trojan tech Inc, 2001.

[23] W. Zhang and J.C. Tu. Effect of ultraviolet disinfection of hydroponic solutions on pythium root rot and non-target bacteria. European journal of plant pathology, 106(5):415-421, 2000.

[24] K. Zhou and A. J.C. Doyle. Essentials of robust control. Prentice Hall, 1997. 